O.C. Zienkiewicz

Department of Civil Engineering University College of Swansea Swansea SA2 8PP, U.K.

Maosong Huang

Department of Civil Engineering Zhejiang University Hangzhou 310027, P.R. China

Jie Wu

Department of Civil Engineering University College of Swansea Swansea SA2 8PP, U.K.

Shiming Wu

Department of Civil Engineering Zhejiang University Hangzhou 310027, P.R. China

\title{
A New Algorithm for the Coupled Soil-Pore Fluid Problem
}

Two new semiexplicit algorithms for the coupled soil-pore fluid problem are developed in this article. The stability of the new algorithms is much better than that of the previous algorithm. The first new scheme ( $\mathbf{H}^{*}$-scheme) based on operator splitting before spatial discretization can avoid the restriction of mixed formulation in the incompressible (zero permeability) limit. The steady-state formulation is discussed to verify this argument. Several examples illustrate the article. (c) 1993 John Wiley \& Sons, Inc.

\section{INTRODUCTION}

The solution of problems in which coupling occurs between the displacement of the soil skeleton (characterized by the displacement $u_{i}$ ) and the pore fluid motion (characterized by the pressure $p$ ) is fundamental in the treatment of engineering designs in which consolidation or dynamic (earthquake) actions occurs. The basis of the treatment is discussed fully by Zienkiewicz and Shiomi [1984] and more recently by Zienkiewicz, Chan, Pastor, Paul, and Shiomi [1990] who deal with many situations involving soil degradation in dynamics. However difficulties exist.

(a) The formulation requires the satisfaction of the so-called Babuska-Brezzi (BB) con- ditions in the limit of nearly incompressible pore fluid and small permeability. This leads to nonuniform interpolation of the $u_{i}$ and $p$ variable as shown by Zienkiewicz et al. [1990] that complicates coding and results in an inconvenient form of semiexplicit or explicit algorithms.

(b) If a semiexplicit or explicit time stepping algorithm is used as first suggested by Zienkiewicz, Hinton, Leung, and Taylor [1980], the time step is controlled by the Courant number based on sound velocity in the undrained medium and on the permeability. This results in uneconomically small time steps needed for a practical solution. Similar limitations exist in the formulation where in place of $p$ the relative 
velocity of porous fluid $w_{i}$ is used as discussed in Zienkiewicz and Shiomi [1984] and more recently implemented by Chan, Famiyesin, and Wood [1991].

For these reasons we introduce here an alternative, staggered, formulation that

(i) permits equal interpolation (continuous interpolation) to be used for $u_{i}$ and $p$; and

(ii) in its semiexplicit form is governed by time steps involving only the sound speed in the drained soil. Such time steps are of course at least one order of magnitude greater than in the previous algorithm. The present work is motivated by somewhat similar forms of transient solution introduced for the study of fluid mechanics with small compressibility by Zienkiewicz and $\mathrm{Wu}$ [1991].

\section{Governing Equations and New Algorithm}

The equations of coupled soil skeleton and pore fluid interaction are derived in Zienkiewicz and Shiomi [1984] and Zienkiewicz et al. [1990]. These can be written as

$$
\begin{gathered}
\mathbf{S}^{T}\left(\boldsymbol{\sigma}^{\prime}-\mathbf{m} p\right)+\rho \mathbf{b}-\rho \ddot{\mathbf{u}}=0 \\
\mathbf{m}^{T} \mathbf{S} \dot{\mathbf{u}}-\nabla^{T} \bar{k} \nabla p+\frac{n}{K_{f}} \dot{p}+\nabla^{T} \bar{k} \rho_{f} \mathbf{b}=\mathbf{0}
\end{gathered}
$$

where $\mathbf{u}$ is the vector of displacement, $p$ is the fluid pressure, $\mathbf{b}$ is the body force per unit mass, $\rho$ is the total density of the mixture, $\rho_{f}$ is the density of the fluid, $n$ is the porosity, $\bar{k}=k / \rho_{f} g$ is the dynamic permeability, $g$ is the gravity acceleration, $K_{f}$ is the bulk modulus of the fluid and $\boldsymbol{\sigma}^{\prime}$ is the vector form of the effective stress defined as $\boldsymbol{\sigma}^{\prime}=\boldsymbol{\sigma}+\mathbf{m} p$ (with $\boldsymbol{\sigma}$ being total stress with the coefficient $\alpha=1$ [Zienkiewicz and Shiomi, 1984]). The strain operator $\mathbf{S}$ and vector $\mathbf{m}$ are given for two dimension $(x, y)$ as

$$
\begin{gathered}
\mathbf{S}=\left[\begin{array}{cc}
\frac{\partial}{\partial x} & 0 \\
0 & \frac{\partial}{\partial y} \\
\frac{\partial}{\partial y} & \frac{\partial}{\partial x}
\end{array}\right] \\
\mathbf{m}^{T}=[1,1,0] .
\end{gathered}
$$

Equation (1) is that of total momentum equilibrium equation and Eq. (2) governs the flow of the fluid in the pores. The equations have to be supplemented by an incremental constitutive law for the soil skeleton linking $\boldsymbol{\sigma}^{\prime}$ and the strain

$$
\varepsilon=\text { Su }
$$

In general for any nonlinear materials we shall consider an incremental form with a tangential modulus $\mathbf{D}$ dependent on $\boldsymbol{\sigma}^{\prime}$ etc.

$$
d \boldsymbol{\sigma}^{\prime}=\mathbf{D} d \boldsymbol{\varepsilon} .
$$

However, in some of the examples we shall assume elastic behavior with $\mathbf{D}$ being constant. Boundary conditions for the problem are generally specified as follows,

$$
\begin{gathered}
u_{i}=\tilde{u}_{i} \text { on } \Gamma_{u} \\
\text { or } t_{i}=\sigma_{i j}^{\prime} n_{j}-n_{i} p=\tilde{t}_{i} \quad \text { on } \Gamma_{t}
\end{gathered}
$$

and

$$
\begin{gathered}
p=\tilde{p} \quad \text { on } \Gamma_{p} \\
\text { or } \quad \bar{k} \frac{\partial p}{\partial n}=-\tilde{q} \quad \text { on } \Gamma_{q}
\end{gathered}
$$

To proceed with the development of the present algorithm, we shall first discretize the governing equations in time only using the GNpj algorithm [Katona and Zienkiewicz, 1985; Zienkiewicz et al. 1990]. Thus for u we shall use GN22 (essentially the Newmark method), writing

$$
\begin{gathered}
\mathbf{u}_{n+1}=\mathbf{u}_{n}+\Delta t \dot{\mathbf{u}}_{n}+\frac{1}{2} \Delta t^{2} \ddot{\mathbf{u}}_{n}+\beta \Delta t^{2} \Delta \ddot{\mathbf{u}}_{n} \\
=\mathbf{u}_{n+1}^{p}+\beta \Delta t^{2} \Delta \ddot{\mathbf{u}}_{n} \\
\dot{\mathbf{u}}_{n+1}=\dot{\mathbf{u}}_{n}+\Delta t \ddot{\mathbf{u}}_{n}+\gamma \Delta t \Delta \ddot{\mathbf{u}}_{n}=\dot{\mathbf{u}}_{n+1}^{p}+\gamma \Delta t \Delta \ddot{\mathbf{u}}_{n} \\
\ddot{\mathbf{u}}_{n+1}=\ddot{\mathbf{u}}_{n}+\Delta \ddot{\mathbf{u}}_{n}=\ddot{\mathbf{u}}_{n+1}^{p}+\Delta \ddot{\mathbf{u}}_{n}
\end{gathered}
$$

For the variables $p$ that occur in first order we shall use GN11 as

$$
\begin{gathered}
p_{n+1}=p_{n}+\Delta t \dot{p}_{n}+\theta \Delta t \Delta \dot{p}_{n}=p_{n+1}^{p}+\theta \Delta t \Delta \dot{p}_{n} \\
\dot{p}_{n+1}=\dot{p}_{n}+\Delta \dot{p}_{n}=\dot{p}_{n+1}^{p}+\Delta \dot{p}_{n} .
\end{gathered}
$$

In the above $\mathbf{u}_{n+1}^{p}$, etc., stand for values that are 
predicted from known parameters at time $t_{n}$ and $\Delta \ddot{\mathbf{u}}_{n}, \Delta \dot{p}_{n}$ are the unknowns. Equation (1) written at $t=t_{n+1}$ is

$$
\mathbf{S}^{T}\left(\boldsymbol{\sigma}_{n+1}^{\prime}-\mathbf{m} p_{n+1}\right)+\rho \mathbf{b}_{n+1}-\rho \ddot{\mathbf{u}}_{n+1}=0 .
$$

In the above equation we shall use the explicit scheme $(\beta=0)$. Thus we write

$$
\mathbf{S}^{T}\left[\boldsymbol{\sigma}_{n+1}^{\prime p}-\mathbf{m}\left(p_{n}+\Delta t \dot{p}_{n}+\Delta \dot{p}_{n} \theta \Delta t\right)\right]
$$$$
+\rho \mathbf{b}_{n+1}-\rho\left(\ddot{\mathbf{u}}_{n+1}^{p}+\Delta \ddot{\mathbf{u}}_{n}\right)=0
$$

where the "predicted" $\boldsymbol{\sigma}_{n+1}^{\prime p}$ is obtained from $\mathbf{u}_{n+1}^{p}$ that takes as

$$
\mathbf{u}_{n+1}^{p}=\mathbf{u}_{n}+\Delta t \dot{\mathbf{u}}_{n}+\frac{1}{2} \Delta t^{2} \ddot{\mathbf{u}}_{n} .
$$

The unknown $\Delta \ddot{\mathbf{u}}_{n}$ can conveniently be separated into two parts:

$$
\Delta \ddot{\mathbf{u}}_{n}=\Delta \ddot{\mathbf{u}}_{n}^{*}+\Delta \ddot{\mathbf{u}}_{n}^{* *} .
$$

We now introduce an operator split of the Eq. (9), and we write this as two equations

$$
\begin{gathered}
\mathbf{S}^{T}\left(\boldsymbol{\sigma}_{n+1}^{p}-\mathbf{m} p_{n}\right)+\rho \mathbf{b}_{n+1}-\rho\left(\ddot{\mathbf{u}}_{n+1}^{p}+\Delta \ddot{\mathbf{u}}_{n}^{*}\right)=0 \\
-\rho \Delta \ddot{\mathbf{u}}_{n}^{* *}-\mathbf{S}^{T} \mathbf{m}\left(\Delta t \dot{p}_{n}+\Delta \dot{p}_{n} \theta \Delta t\right)=0
\end{gathered}
$$

Equation (12) contains only one independent unknown, which we shall find useful later. Similarly with an implicit time discretization, Eq. (2) is written at $t=t_{n+1}$ as

$$
\begin{aligned}
\mathbf{m}^{T} \mathbf{S}\left(\dot{\mathbf{u}}_{n+1}^{p}\right. & \left.+\Delta \ddot{\mathbf{u}}_{n}^{*} \gamma \Delta t+\Delta \ddot{\mathbf{u}}_{n}^{* *} \gamma \Delta t\right) \\
& -\nabla^{T} \bar{k} \nabla\left(p_{n+1}^{p}+\theta \Delta t \Delta \dot{p}_{n}\right) \\
& +\frac{n}{K_{f}}\left(\dot{p}_{n+1}^{p}+\Delta \dot{p}_{n}\right)+\nabla^{T} \bar{k} \rho_{f} \mathbf{b}_{n+1}=0 .
\end{aligned}
$$

By substituting Eq. (13) into Eq. (14), we can eliminate the unknown $\Delta \ddot{\mathbf{u}}_{n}^{* *}$ and now have

$$
\begin{aligned}
\mathbf{m}^{T} \mathbf{S}\left[\dot{\mathbf{u}}_{n+1}^{p}\right. & +\Delta \ddot{\mathbf{u}}_{n}^{*} \gamma \Delta t \\
& \left.-\frac{1}{\rho} \mathbf{S}^{T} \mathbf{m}\left(\Delta t \dot{p}_{n}+\Delta \dot{p}_{n} \theta \Delta t\right) \gamma \Delta t\right] \\
& -\nabla^{T} \bar{k} \nabla\left(p_{n+1}^{p}+\theta \Delta t \Delta \dot{p}_{n}\right) \\
& +\frac{n}{K_{f}}\left(\dot{p}_{n+1}^{p}+\Delta \dot{p}_{n}\right)+\nabla^{T} \bar{k} \rho_{f} \mathbf{b}_{n+1}=0
\end{aligned}
$$

At this stage we perform the spatial discretization and apply the standard Galerkin procedure to the Eqs. (12), (15), and (13). We can easily obtain the following algebraic equations:

$$
\begin{gathered}
\mathbf{M}\left(\ddot{\overline{\mathbf{u}}}_{n+1}^{p}+\Delta \ddot{\overline{\mathbf{u}}}_{n}^{*}\right)+\left(\int_{\Omega} \mathbf{B}^{T} \mathbf{\sigma}^{\prime} d \Omega\right)_{n+1}^{p} \\
-\mathbf{Q} \overline{\mathbf{p}}_{n}=\left(\mathbf{f}_{s}^{*}\right)_{n+1} \\
\left(\mathbf{S}+\mathbf{H} \theta \Delta t+\mathbf{H}^{*} \theta \gamma \Delta t^{2}\right) \Delta \dot{\overline{\mathbf{p}}}_{n}=\left(\mathbf{f}_{p}\right)_{n+1}-\mathbf{H}^{*} \dot{\overline{\mathbf{p}}}_{n} \gamma \Delta t^{2} \\
-\mathbf{Q}^{T}\left(\dot{\overline{\mathbf{u}}}_{n+1}^{p}+\Delta \ddot{\overline{\mathbf{u}}}_{n}^{*} \gamma \Delta t\right)-\mathbf{S} \dot{\overline{\mathbf{p}}}_{n+1}^{p}-\mathbf{H} \overline{\mathbf{p}}_{n+1}^{p} \\
\mathbf{M} \Delta \ddot{\overline{\mathbf{u}}}_{n}^{* *}=\mathbf{Q}\left(\Delta t \dot{\overline{\mathbf{p}}}_{n}+\Delta \dot{\overline{\mathbf{p}}}_{n} \theta \Delta t\right)+\left(\mathbf{f}_{s}^{* *}\right)_{n+1}
\end{gathered}
$$

where $\mathbf{u}=\mathbf{N}_{u} \overline{\mathbf{u}}, p=\mathbf{N}_{p} \overline{\mathbf{p}}$, etc., and

$$
\begin{gathered}
\mathbf{M}=\int_{\Omega} \mathbf{N}_{u}^{T} \rho \mathbf{N}_{u} d \Omega \\
\mathbf{Q}=\int_{\Omega} \mathbf{B}^{T} \mathbf{m} \mathbf{N}_{p} d \Omega \\
\mathbf{H}=\int_{\Omega}\left(\nabla \mathbf{N}_{p}\right)^{T} \bar{k}\left(\nabla \mathbf{N}_{p}\right) d \Omega \\
\mathbf{H}^{*}=\int_{\Omega}\left(\nabla \mathbf{N}_{p}\right)^{T} \frac{1}{\rho}\left(\nabla \mathbf{N}_{p}\right) d \Omega \\
\mathbf{S}=\int_{\Omega} \mathbf{N}_{p}^{T} \frac{n}{K_{f}} \mathbf{N}_{p} d \Omega \\
\left(\mathbf{f}_{s}^{*}\right)_{n+1}=\int_{\Omega} \mathbf{N}_{u}^{T} \rho \mathbf{b}_{n+1} d \Omega \\
+\int_{\Gamma} \mathbf{N}_{u}^{T} \tilde{\mathbf{t}}_{n+1}^{\prime} d \Gamma-\int_{\Gamma} \mathbf{N}_{u}^{T} \mathbf{n} p_{n} d \Gamma \\
\left(t_{i}^{\prime}=\sigma_{i j}^{\prime} n_{j}=\tilde{t}_{i}^{\prime}\right) \\
\left(\mathbf{f}_{p}\right)_{n+1}=\int_{\Gamma} \mathbf{N}_{p}^{T} \bar{k} \frac{\partial p_{n+1}}{\partial n} d \Gamma \\
+\int_{\Omega}\left(\nabla \mathbf{N}_{p}\right)^{T} \bar{k} \rho_{f} \mathbf{b}_{n+1} d \Omega+\left(\int_{\Gamma} \mathbf{N}_{p}^{T} \frac{1}{\rho} \frac{\partial p_{2}}{\partial n} d \Gamma\right) \gamma \Delta t \\
\left(p_{2}=\Delta t \dot{p}_{n}+\Delta \dot{p}_{n} \theta \Delta t\right) \\
\left(\mathbf{f}_{s}^{* *}\right)_{n+1}=-\int_{\Gamma} \mathbf{N}_{u}^{T} \mathbf{n} p_{2} d \Gamma \\
\left.\left(\int_{\Omega} \mathbf{B}^{\mathbf{T}} \boldsymbol{\sigma}^{\prime} d \Omega\right)_{n+1}^{p}=\mathbf{P}_{n} \mathbf{u}_{n+1}^{p}\right) \\
=\int_{\Omega} \mathbf{B}^{T}\left(\Delta \mathbf{\sigma}_{n}^{\prime}\right)^{p} d \Omega+\mathbf{P}\left(\mathbf{u}_{n}\right) .
\end{gathered}
$$

$\left(\Delta \boldsymbol{\sigma}_{n}^{\prime}\right)^{p}$ is related to $\Delta \mathbf{u}_{n}^{p}$ that is defined as follows,

$$
\Delta \mathbf{u}_{n}^{p}=\mathbf{u}_{n+1}^{p}-\mathbf{u}_{n} .
$$


The solution can proceed now as follows:

Step 1: solve $\Delta \ddot{\overline{\mathbf{u}}}_{n}^{*}$ using Eq. (16).

Step 2: solve $\Delta \dot{\overline{\mathbf{p}}}_{\text {}}$ using Eq. (17).

Step 3: solve $\Delta \ddot{\overline{\mathbf{u}}}_{n}^{* *}$ using Eq. (18).

Step 4: compute $\Delta \ddot{\overline{\mathbf{u}}}_{n}=\Delta \ddot{\overline{\mathbf{u}}}_{n}^{*}+\Delta \ddot{\overline{\mathbf{u}}}_{n}^{* *}$ and determine $\overline{\mathbf{u}}_{n+1}, \overline{\mathbf{p}}_{n+1}$, etc.

If the algorithm is used in a semiimplicit form with $\theta \geq \frac{1}{2}$ (in our examples $\theta=\frac{1}{2}$ is taken) then an explicit type of computation can be used in step 1 and 3 requiring only the inversion of the matrix $\mathbf{M}$ that can be made trivial by lumping. In that case the stability is conditional but is much better than that of Zienkiewicz et al.'s scheme [1980], especially for small compressibility and small permeability. Indeed the critical time step is independent on the permeability and compressibility. With $\gamma=0.5$, the critical time step can be written for linear elements as

$$
\Delta t_{\text {crit }}=\frac{h}{c_{d}}
$$

where $h$ is the element size and $c_{\mathrm{d}}$ is the speed of elastic wave propagation in the drained soil. The stability of the new schemes is identical to that of the explicit-implicit direct scheme of Zienkiewicz et al. [1990].

For both semiexplicit and explicit versions we shall find by using precisely the same arguments as in Zienkiewicz and Wu [1980] that the limitations of BB condition are avoided. In the examples given later we shall see this demonstration. Appendix A fully discusses the reasons for this phenomenon.

\section{AN ALTERNATIVE STAGGERED SCHEME (AFTER SPATIAL DISCRETIZATION)}

A similar operator splitting scheme can also be applied after spatial discretization. Here we use the spatial approximation in the form

$$
\begin{aligned}
& \mathbf{u} \approx \mathbf{u}(t)=\mathbf{N}_{u} \overline{\mathbf{u}}(t) \\
& p \approx p(t)=\mathbf{N}_{p} \overline{\mathbf{p}}(t)
\end{aligned}
$$

and by the use of an appropriate Galerkin statements for Eqs. (1) and (2), we have the following finite element discrete approximation:

$$
\mathbf{M} \ddot{\overline{\mathbf{u}}}+\int_{\Omega} \mathbf{B}^{T} \sigma^{\prime} d \Omega-\mathbf{Q} \overline{\mathbf{p}}=\mathbf{f}_{s}
$$

and

$$
\mathbf{Q}^{T} \dot{\overline{\mathbf{u}}}+\mathbf{H} \overline{\mathbf{p}}+\mathbf{S} \dot{\overline{\mathbf{p}}}=\mathbf{f}_{p}
$$

where

$$
\begin{gathered}
\mathbf{f}_{s}=\int_{\Omega} \mathbf{N}_{u}^{T} \rho \mathbf{b} d \Omega+\int_{\Omega} \mathbf{N}_{u}^{T} \tilde{\mathbf{t}} d \Gamma \\
\mathbf{f}_{p}=\int_{\Gamma} \mathbf{N}_{p}^{T} \bar{k} \frac{\partial p}{\partial n} d \Gamma+\int_{\Omega}\left(\nabla \mathbf{N}_{p}\right)^{T} \bar{k} \rho_{f} \mathbf{b} d \Omega
\end{gathered}
$$

Again we shall use GN22, with $\beta=0$, and obtain

$$
\begin{aligned}
\mathbf{M}\left(\ddot{\mathbf{u}}_{n+1}^{p}\right. & \left.+\Delta \ddot{\overline{\mathbf{u}}}_{n}\right)+\left(\int_{\Omega} \mathbf{B}^{T} \boldsymbol{\sigma}^{\prime} d \Omega\right)_{n+1}^{p} \\
& -\mathbf{Q}\left(\overline{\mathbf{p}}_{n}+\Delta t \dot{\overline{\mathbf{p}}_{n}}+\Delta \dot{\overline{\mathbf{p}}}_{n} \theta \Delta t\right)+\left(\mathbf{f}_{s}\right)_{n+1}
\end{aligned}
$$

and similarly

$$
\begin{aligned}
\mathbf{Q}^{T}\left(\dot{\overline{\mathbf{u}}}_{n+1}^{p}\right. & \left.+\Delta \ddot{\overline{\mathbf{u}}}_{n} \gamma \Delta t\right)+\mathbf{S}\left(\dot{\overline{\mathbf{p}}}_{n+1}^{p}+\Delta \dot{\overline{\mathbf{p}}}_{n}\right) \\
& +\mathbf{H}\left(\mathbf{p}_{n+1}^{p}+\Delta \dot{\overline{\mathbf{p}}}_{n} \theta \Delta t\right)=\left(\mathbf{f}_{p}\right)_{n+1} .
\end{aligned}
$$

Introducing the previous splitting scheme and following the same steps, we can easily obtain in place of Eqs. (16)-(18) the following

$$
\begin{gathered}
\mathbf{M}\left(\ddot{\overline{\mathbf{u}}}_{n+1}^{p}+\Delta \ddot{\overline{\mathbf{u}}}_{n}^{*}\right) \\
\quad+\left(\int_{\Omega} \mathbf{B}^{T} \boldsymbol{\sigma}^{\prime} d \Omega\right)_{n+1}^{p}-\mathbf{Q} \overline{\mathbf{p}}_{n}=\left(\mathbf{f}_{s}\right)_{n+1} \\
\left(\mathbf{S}+\mathbf{H} \theta \Delta t+\mathbf{Q}^{T} \mathbf{M}^{-1} \mathbf{Q} \theta \gamma \Delta t^{2}\right) \Delta \dot{\overline{\mathbf{p}}}_{n} \\
=\left(\mathbf{f}_{p}\right)_{n+1}-\mathbf{Q}^{T} \mathbf{M}^{-1} \mathbf{Q} \dot{\overline{\mathbf{p}}}_{n} \gamma \Delta t^{2} \\
-\mathbf{Q}^{T}\left(\dot{\mathbf{u}}_{n+1}^{p}+\Delta \ddot{\overline{\mathbf{u}}}_{n}^{*} \gamma \Delta t\right)-\mathbf{S} \dot{\overline{\mathbf{p}}}_{n+1}^{p}-\mathbf{H} \overline{\mathbf{p}}_{n+1}^{p} \\
\mathbf{M} \Delta \ddot{\overline{\mathbf{u}}}_{n}^{* *}=\mathbf{Q}\left(\Delta t \dot{\overline{\mathbf{p}}}_{n}+\Delta \dot{\overline{\mathbf{p}}}_{n} \theta \Delta t\right) .
\end{gathered}
$$

Although Eqs. (23) and (25) are identical to Eqs. (16) and (18), Eq. (24) is only similar to Eq. (17). Here the matrix $\mathbf{Q}^{T} \mathbf{M}^{-1} \mathbf{Q}$ with a wider connection replaces the $\mathbf{H}^{*}$ matrix and its solution is not trivial in the large problem. For this reason we use a preconditioned conjugate gradient (PCG) iterative solver with diagonal preconditioner

$$
\mathbf{B}=\operatorname{diag}\left(\mathbf{S}+\mathbf{H} \theta \Delta t+\mathbf{Q}^{T} \mathbf{M}^{-1} \mathbf{Q} \theta \gamma \Delta t^{2}\right) .
$$

For the linear equation $\mathbf{A X}=\mathbf{b}$, the general PCG iterative procedure has been described in Borja [1991]. The flowchart in Table 1 summarizes the steps necessary to carry out the solution of a linear system arising out of Newton's iteration by the PCG method. By using this iterative solver, we shall also get an economic solution on most large computations.

Even though the stability of this alternative scheme ( $\mathbf{Q}^{T} \mathbf{M}^{-1} \mathbf{Q}$-scheme) is the same as that of 
Table 1. Flowchart of the Preconditioned Conjugate Gradient Algorithm

1. Initialize $k=0, \quad x^{0}=\mathbf{0}, \quad r^{0}=b, \quad p^{0}=z^{0}=$ $\boldsymbol{B}^{-1} \boldsymbol{r}^{0}$

2. $\alpha^{k}=\left\langle\mathbf{r}^{k}, \boldsymbol{z}^{k}\right\rangle /\left\langle\boldsymbol{p}^{k}, \boldsymbol{A} \boldsymbol{p}^{k}\right\rangle$

3. $\boldsymbol{x}^{k+1}=\boldsymbol{x}^{k}+\alpha^{k} \boldsymbol{p}^{k}$

4. $\boldsymbol{r}^{k+1}=\boldsymbol{r}^{k}-\alpha^{k} \boldsymbol{A} \boldsymbol{p}^{k}$

5. If $\left\|\boldsymbol{r}^{k+1}\right\| /\left\|\boldsymbol{r}^{0}\right\| \leq \mathrm{TOL}$, return; else continue

6. $\boldsymbol{z}^{k+1}=\boldsymbol{B}^{-1} \boldsymbol{r}^{k+1}$

7. $\beta^{k}=-\left\langle z^{k+1}, \boldsymbol{A} \boldsymbol{p}^{k}\right\rangle /\left\langle\boldsymbol{p}^{k}, \boldsymbol{A} \boldsymbol{p}^{k}\right\rangle$

8. $\boldsymbol{p}^{k+1}=z^{k+1}+\beta^{k} \boldsymbol{p}^{k}$

9. $k \leftarrow k+1$ and go to Step 2 .

the first scheme ( $\mathbf{H}^{*}$-scheme), it must be noted that this scheme does not avoid the restriction of mixed formulation as shown in Appendix A. We shall also demonstrate this in the example section.

\section{EXAMPLES}

The following numerical examples are solved to show the performance of the algorithms in various semiexplicit $\left(\beta=0, \theta=\gamma=\frac{1}{2}\right)$ and fully implicit $\left(\beta=\frac{1}{4}, \theta=\gamma=\frac{1}{2}\right)$.

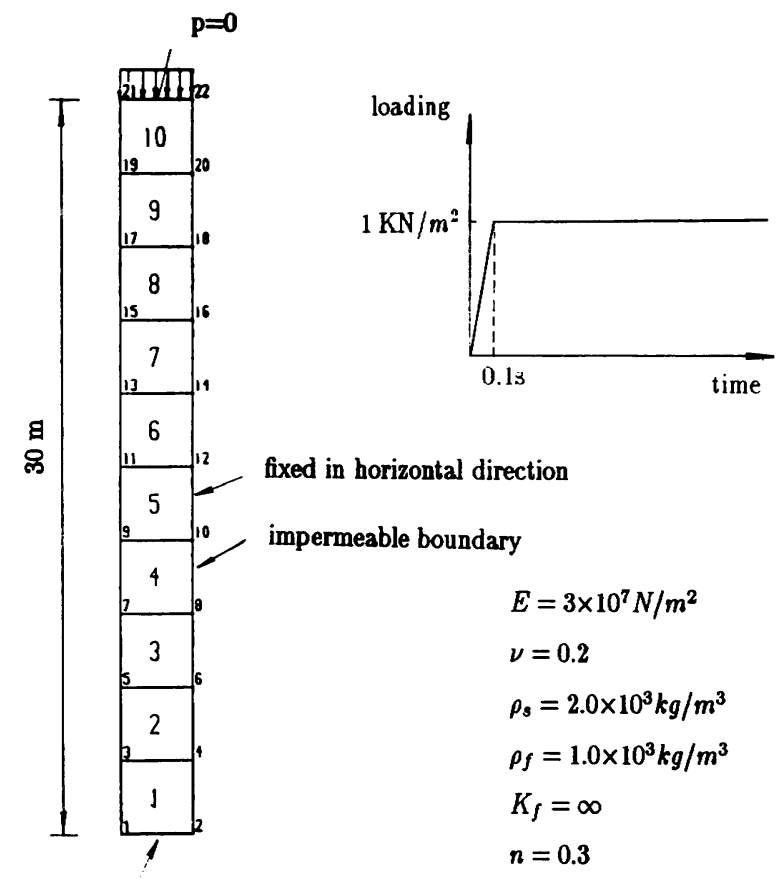

impermeable and fixed boundary

FIGURE 1 Saturated soil column problem.
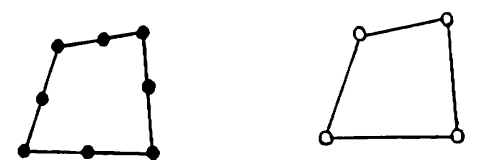

biquadratic for $\mathbf{u}$ and bilinear for $p$

(a) satisfies B.B. condition
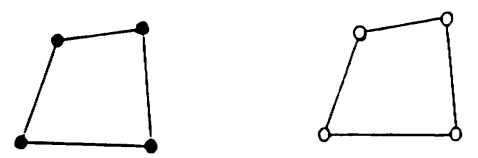

bilinear for $\mathbf{u}$ and bilinear for $p$

(b) violates B.B. condition but OK with new scheme( $H^{*}$-scheme)

FIGURE 2 Elements used for coupled analysis.

\section{Soil Column: Undrained Condition $(k=0)$}

Figure 1 shows the soil column subjected to a surface step loading of $1 \mathrm{KN} / \mathrm{m}^{2}$ applied in time 0.1 seconds. The boundary conditions and the material properties are shown in the figure itself. Incompressible pore fluid is first considered and we consider various permeabilities of soil. Figure 2 shows two forms of the elements used in this paper.

A restriction of mixed formulation exists in the direct implicit scheme to be solved in such a limiting case. This is identical to that used in the solution of problems of incompressible elasticity or fluid mechanics. Such a study places limitations on the approximating function $\mathbf{N}_{u}$ and $\mathbf{N}_{p}$. In Cases $1,2,3$, and 4, time step $\Delta t=0.02 \mathrm{sec}$ onds is used.

Case 1: The calculation is first carried out using the fully implicit scheme of Zienkiewicz et al. [1990] with equal interpolation, that is, bilinear for $\mathbf{u}$ and bilinear for $p$. The computational porepressure values when the steady state is reached are shown in Fig. 3. Obviously, this element form cannot be acceptable at incompressibleundrained limits.

Case 2: Now we use a fully implicit scheme with nonuniform interpolation, that is, biquadratic for $\mathbf{u}$ and bilinear for $p$, that satisfies the $\mathrm{BB}$ condition. The computational results 


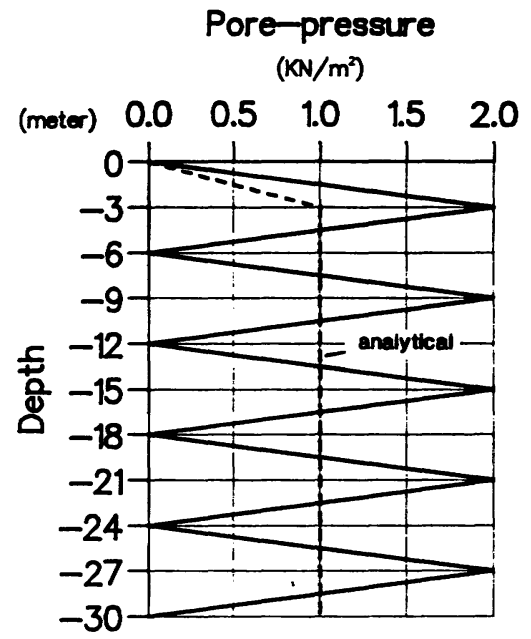

(a) Pore-pressure in the steady state

$\left(\mathrm{KN} / \mathrm{m}^{2}\right)$

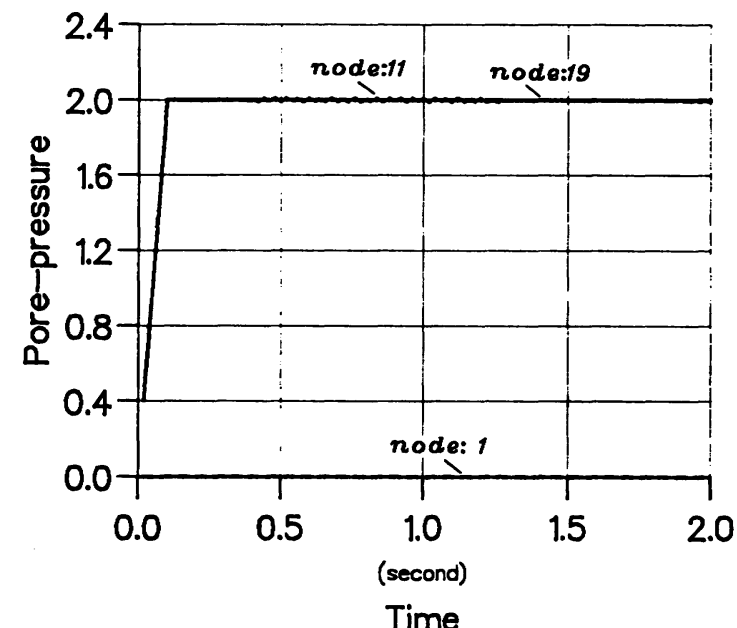

(b) Time history of pore-pressure

FIGURE 3 Solutions for implicit direct scheme with equal interpolation (undrained, incompressible).

shown in Fig. 4 are reasonable and can be used as a basis of comparison.

Case 3: Figure 5 shows numerical results by using a new semiemplicit $\mathbf{H}^{*}$-scheme with equal interpolation (bilinear for $\mathbf{u}$ and bilinear for $p$ ). It is noted that the results are close to those of case 2. As predicted this scheme can avoid restriction of the mixed formulation.

Case 4: We also use a new semiexplicit $\mathbf{Q}^{T} \mathbf{M}^{-1} \mathbf{Q}$-scheme with equal interpolation (bilinear for $\mathbf{u}$ and bilinear for $p$ ) to compute the same example. The results are shown in Fig. 6.
But the result is identical to that in case 1. This scheme of course cannot avoid restriction of the mixed formulation.

We find that $\Delta t=0.022$ seconds leads to instability and the theoretical value of the critical time step $\Delta t_{\text {crit }}=0.0214$ seconds by using new semiexplicit schemes with equal interpolation (bilinear for $\mathbf{u}$ and bilinear for $p$ ). The numerical results show the same limitation of the time step in two new semiexplicit schemes $\left(\mathbf{H}^{*}\right.$-scheme and $\mathbf{Q}^{T} \mathbf{M}^{-1}$ Q-scheme).

Case 5: Further analysis is carried out by the $\mathbf{Q}^{T} \mathbf{M}^{-1} \mathbf{Q}$-scheme with nonuniform interpolation, that is, biquadratic for $\mathbf{u}$ and bilinear for $p$. Time step $\Delta t=0.003$ seconds is used in this case.

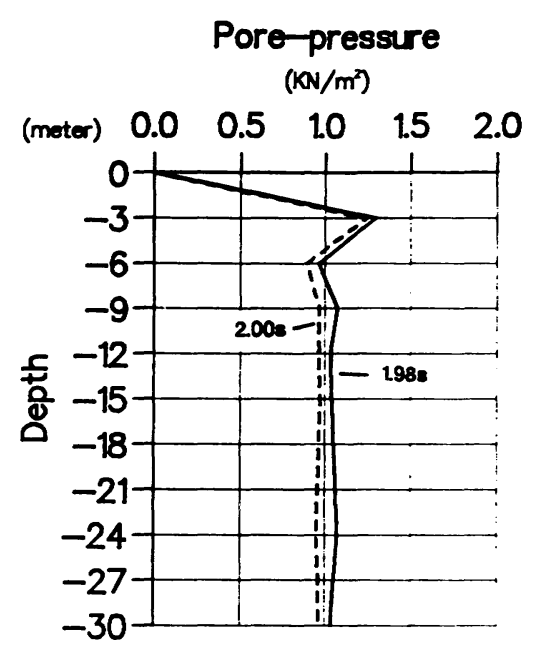

(a) Pore-pressure in the steady state

$\left(\mathrm{KN} / \mathrm{m}^{2}\right)$

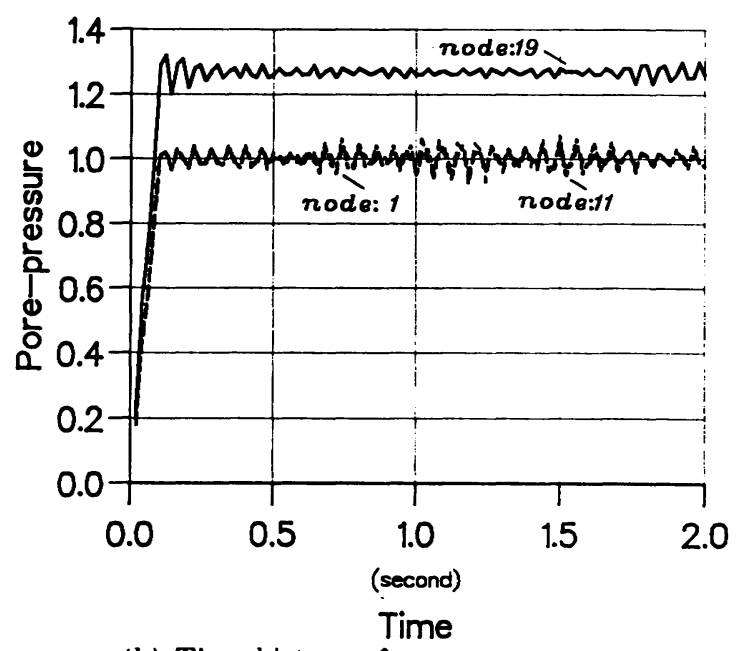

(b) Time history of pore-pressure

FIGURE 4 Solutions for implicit direct scheme with nonuniform interpolation (undrained, incompressible). 
Pore-pressure $\left(\mathrm{KN} / \mathrm{m}^{2}\right)$

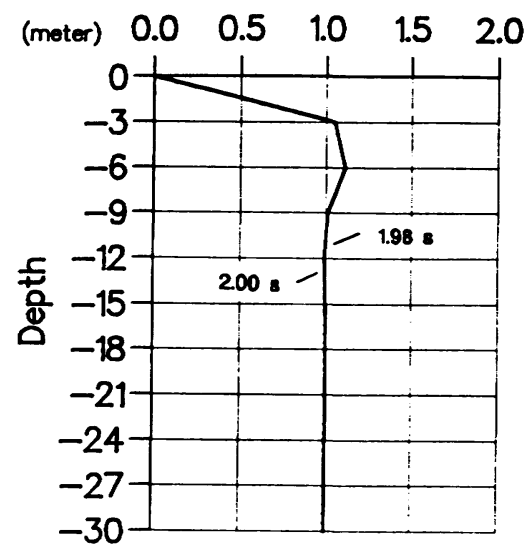

(a) Pore-pressure in the steady state

$\left(\mathrm{KN} / \mathrm{m}^{2}\right)$

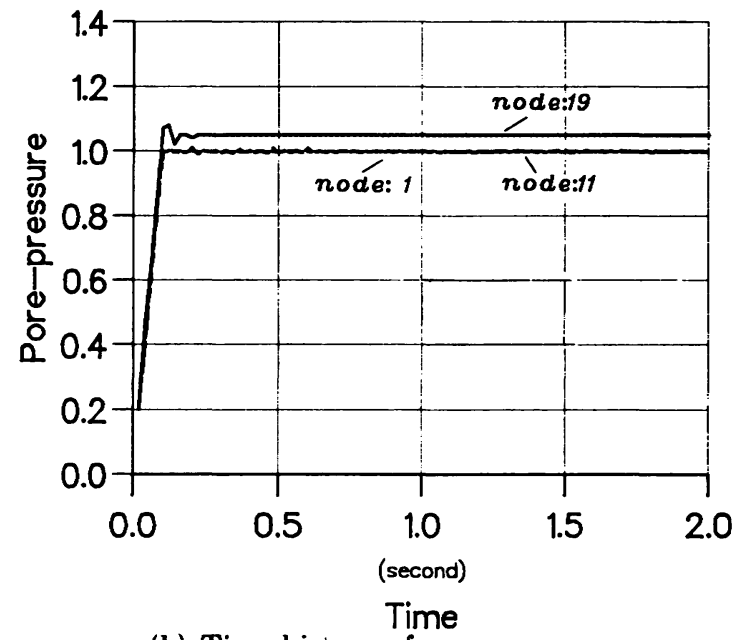

(b) Time history of pore-pressure

FIGURE 5 Solutions for semiexplicit $\mathbf{H}^{*}$-scheme with equal interpolation (undrained, incompressible).

The result shown in Fig. 7 is very close to that in case 2 .

\section{Soil Column: $k=1.0 \times 10^{-4} \mathrm{~m} / \mathrm{s}$}

It is observed that the response of pore pressure in the direct implicit scheme with equal interpolation oscillates significantly and overestimates the initial pore pressure significantly (Fig. 8). The results can be improved when mixed elements are introduced (Fig. 9) or the semiexplicit $\mathbf{H}^{*}$ scheme is adopted (Fig. 10). But we still find that the semiexplicit $\mathbf{Q}^{T} \mathbf{M}^{-1} \mathbf{Q}$-scheme does not perform well (Fig. 11). Time step $\Delta t=0.02$ seconds is used for all schemes.
Soil Column: $k=1.0 \times 10^{-2} \mathrm{~m} / \mathrm{s}$

For large permeability, it seems that every scheme is successful. The results show that the numerical results of two new schemes are very close to those of the direct scheme (Fig. 12), $\mathbf{H}^{*}$ scheme (Fig. 13) and $\mathbf{Q}^{T} \mathbf{M}^{-1} \mathbf{Q}$-scheme (Fig. 14). Time step $\Delta t=0.02$ seconds is used for all schemes.

\section{Soil Foundation: Undrained Condition}

In order to further verify the improvement of the new scheme, we compute a soil foundation with a surface step loading of $1 \mathrm{KN} / \mathrm{m}^{2}$ applied in time

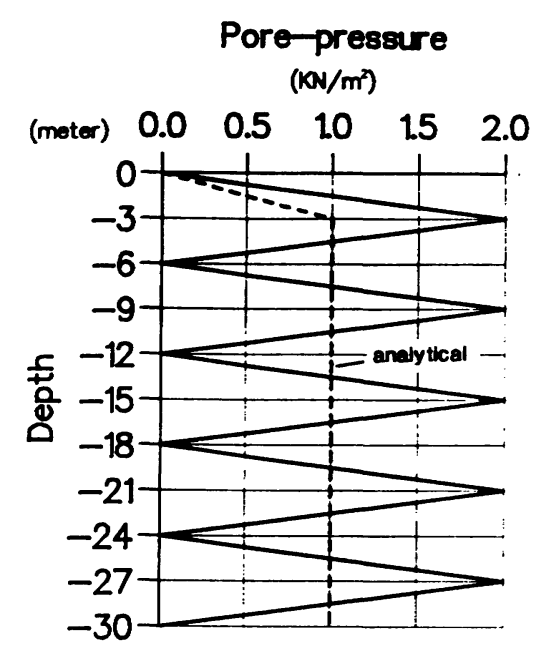

$\left(\mathrm{KN} / \mathrm{m}^{2}\right)$

(a) Pore-pressure in the steady state

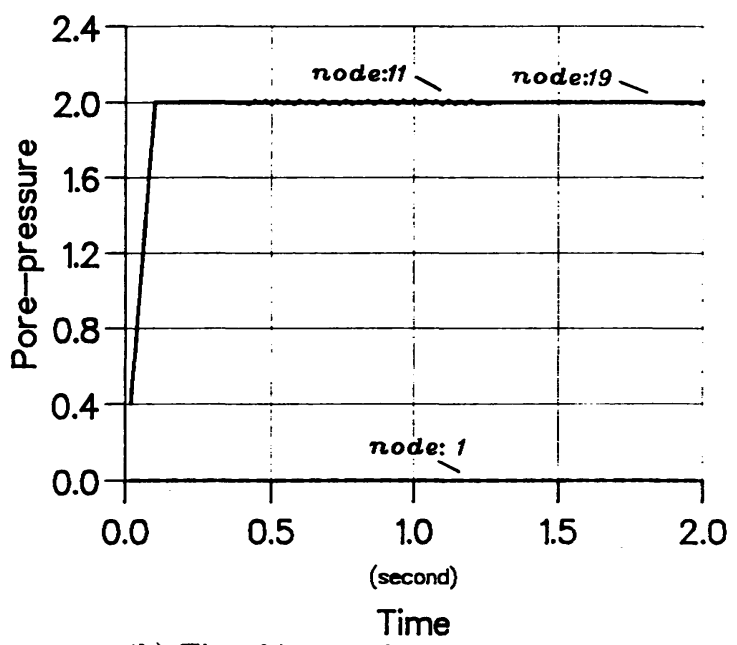

(b) Time history of pore-pressure

FIGURE 6 Solutions for semiexplicit $\mathbf{Q}^{T} \mathbf{M}^{-1} \mathbf{Q}$ scheme with equal interpolation (undrained, incompressible). 


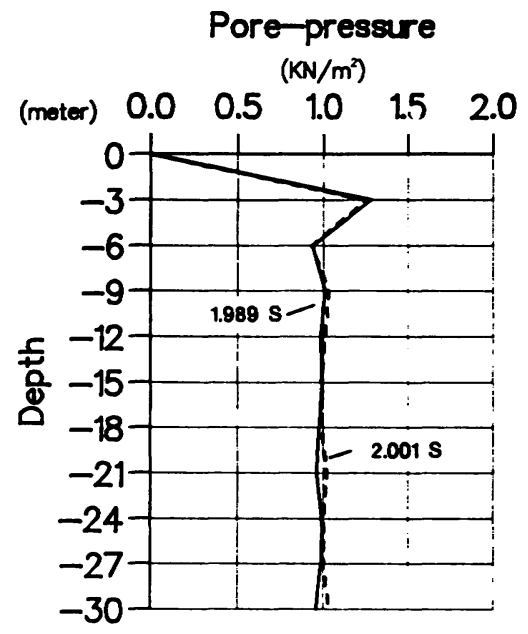

$\left(\mathrm{KN} / \mathrm{m}^{2}\right)$

(a) Pore-pressure in the steady state

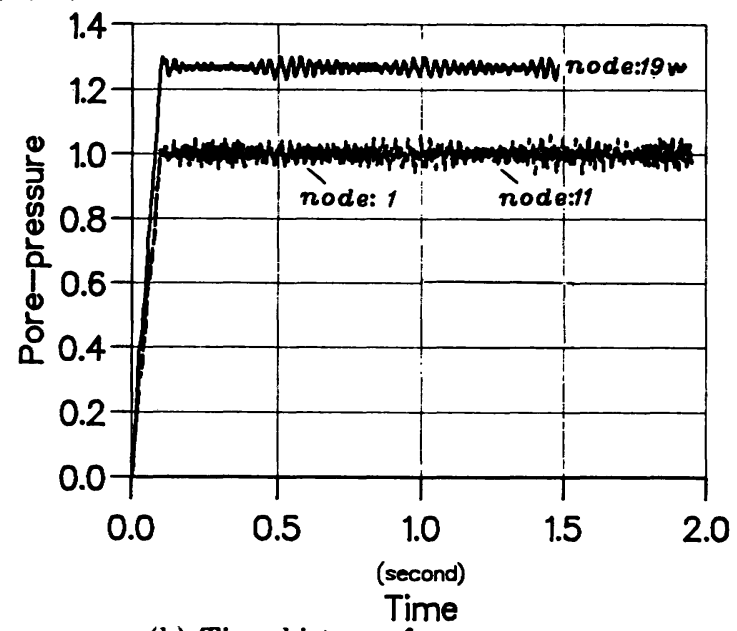

(b) Time history of pore-pressure

FIGURE 7 Solutions for semiexplicit $\mathbf{Q}^{T} \mathbf{M}^{-1} \mathbf{Q}-$ scheme with nonuniform interpolation (undrained, incompressible).

$\left(\mathrm{KN} / \mathrm{m}^{2}\right)$

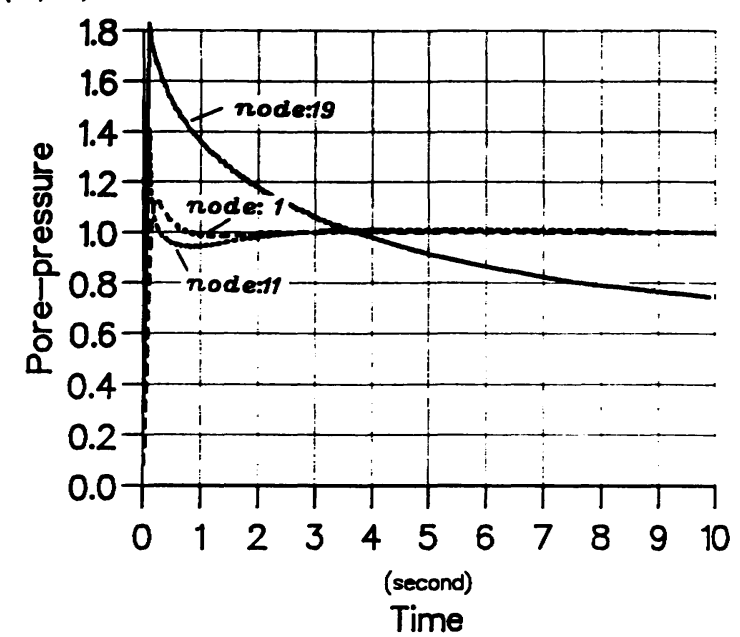

FIGURE 8 Solutions for implicit direct scheme with equal interpolation $\left(k=1.0 \times 10^{-4} \mathrm{~m} / \mathrm{s}\right.$, incompressible).
$\left(\mathrm{KN} / \mathrm{m}^{2}\right)$

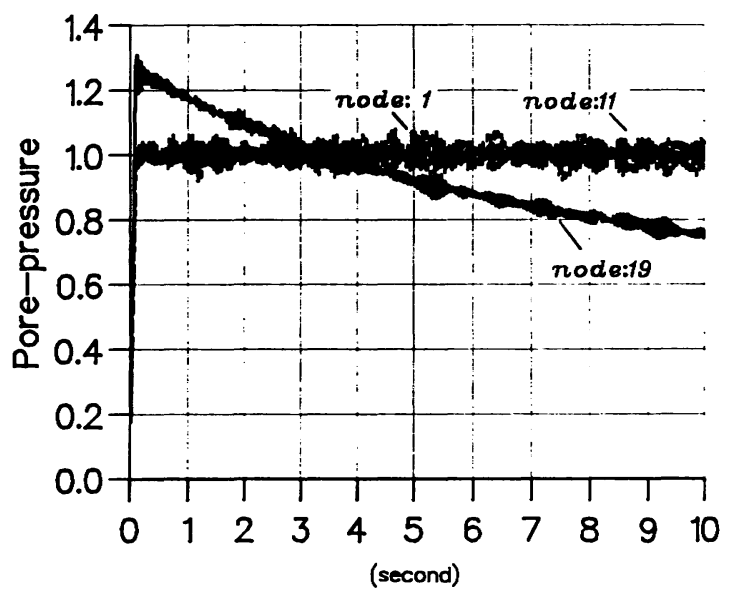

Time

FIGURE 9 Solutions for implicit direct scheme with nonuniform interpolation $\left(k=1.0 \times 10^{-4} \mathrm{~m} / \mathrm{s}\right.$, incompressible).

0.1 seconds (Fig. 15). The boundary conditions are shown in the figure. The material properties are identical to those in example 1. We only consider an incompressible-undrained limit case. The results shown in Figs. 16, 17, 18, and 19 will lead to the same conclusions as those of example 1. Time step $\Delta t=0.02$ seconds is used in this example.

\section{CONCLUSION}

In this paper we have presented two new, staggered, semiexplicit algorithms for the coupled

$\left(\mathrm{KN} / \mathrm{m}^{2}\right)$

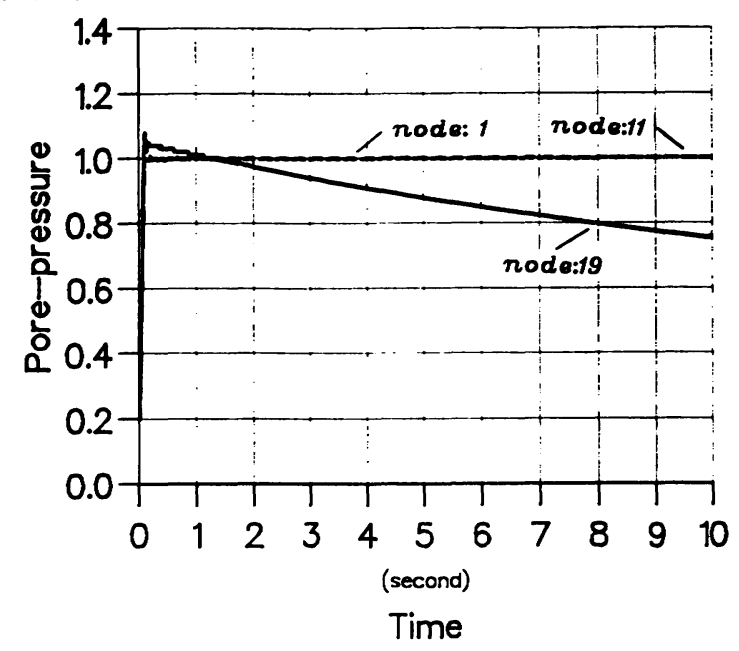

FIGURE 10 Solutions for semiexplicit $\mathbf{H}^{*}$-scheme with equal interpolation $\left(k=1.0 \times 10^{-4} \mathrm{~m} / \mathrm{s}\right.$, incompressible). 
$\left(\mathrm{KN} / \mathrm{m}^{2}\right)$

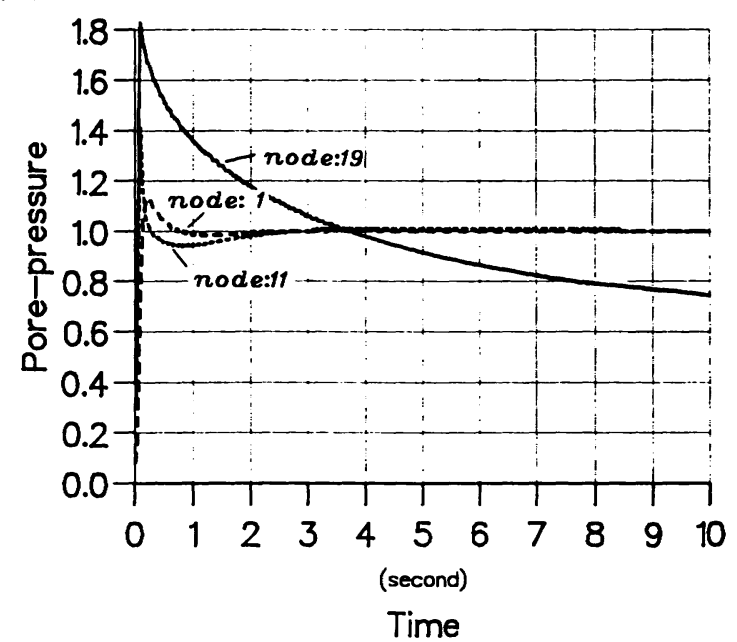

FIGURE 11 Solutions for semiexplicit $\mathbf{Q}^{T} \mathbf{M}^{-1} \mathbf{Q}$ scheme with equal interpolation $\left(k=1.0 \times 10^{-4} \mathrm{~m} / \mathrm{s}\right.$, incompressible).

soil-pore fluid problem. The restriction of mixed formulation can be avoided by the first new scheme ( $\mathbf{H}^{*}$-scheme) based on operator splitting before spatial discretization. The results show that obvious improvement is obtained in the incompressible-undrained limit case. The steadystate solution can be obtained by a time step iterative solver. Though the second new scheme $\left(\mathbf{Q}^{T} \mathbf{M}^{-1} \mathbf{Q}\right.$-scheme) cannot avoid restriction of mixed formulation, it must be noted that the stability of both schemes has considerably improved the limits of Zienkiewicz et al. [1980]. Analysis for more complex elastoplastic dynamic computation will be reported elsewhere. How-

$\left(\mathrm{KN} / \mathrm{m}^{2}\right)$

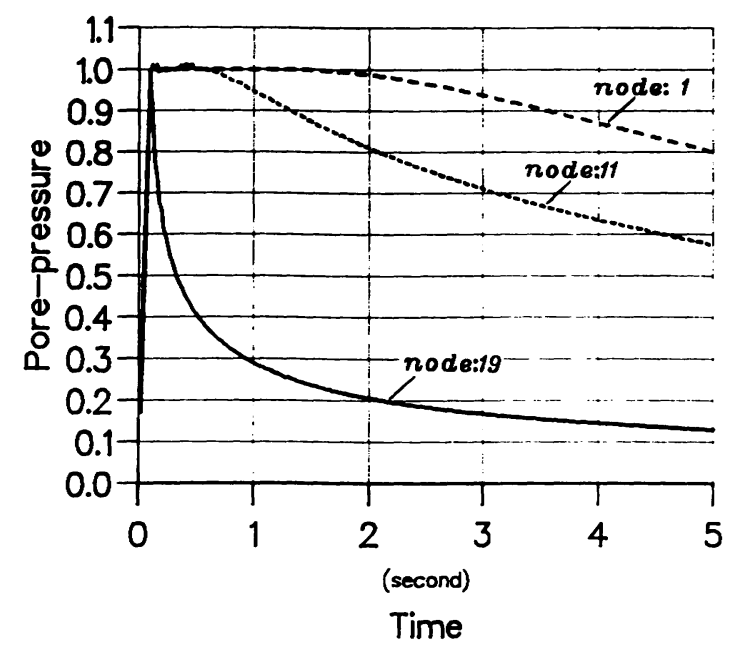

FIGURE 12 Solutions for implicit direct scheme with equal interpolation $\left(k=1.0 \times 10^{-2} \mathrm{~m} / \mathrm{s}\right.$, incompressible).
$\left(\mathrm{KN} / \mathrm{m}^{2}\right)$

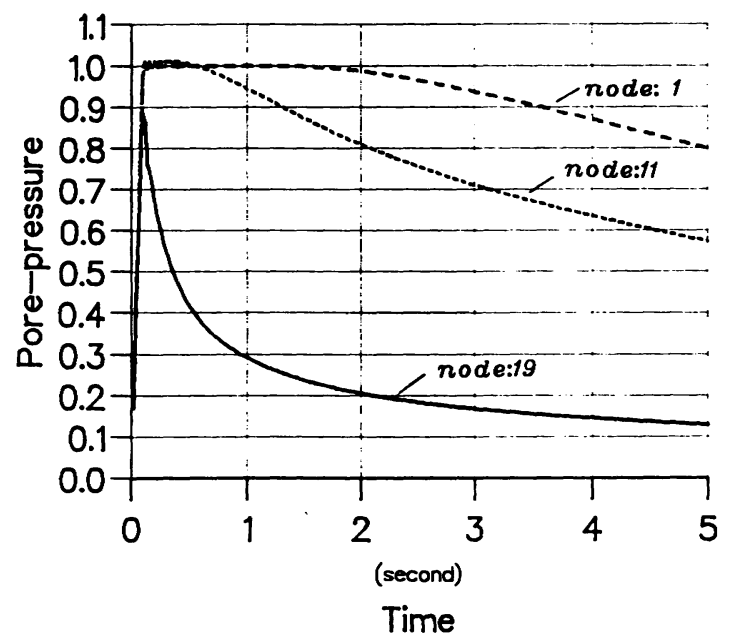

FIGURE 13 Solutions for semiexplicit $\mathbf{H}^{*}$-scheme with equal interpolation $\left(k=1.0 \times 10^{-2} \mathrm{~m} / \mathrm{s}\right.$, incompressible).

$\left(\mathrm{KN} / \mathrm{m}^{2}\right)$

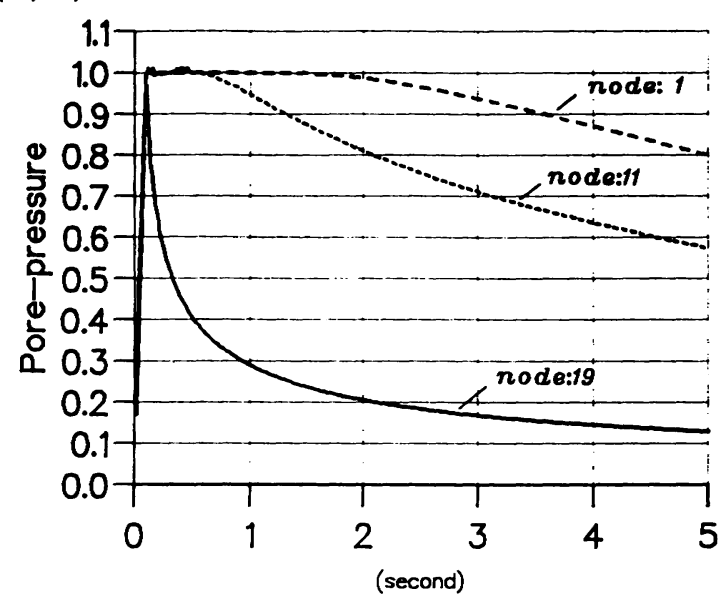

Time

FIGURE 14 Solutions for semiexplicit $\mathbf{Q}^{T} \mathbf{M}^{-1} \mathbf{Q}$ scheme with equal interpolation $\left(k=1.0 \times 10^{-2} \mathrm{~m} / \mathrm{s}\right.$, incompressible).

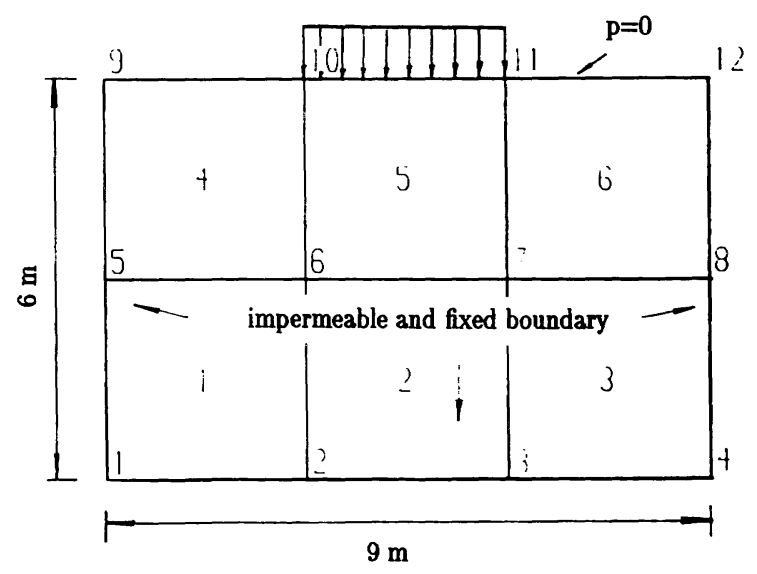

FIGURE 15 Saturated soil foundation problem. 


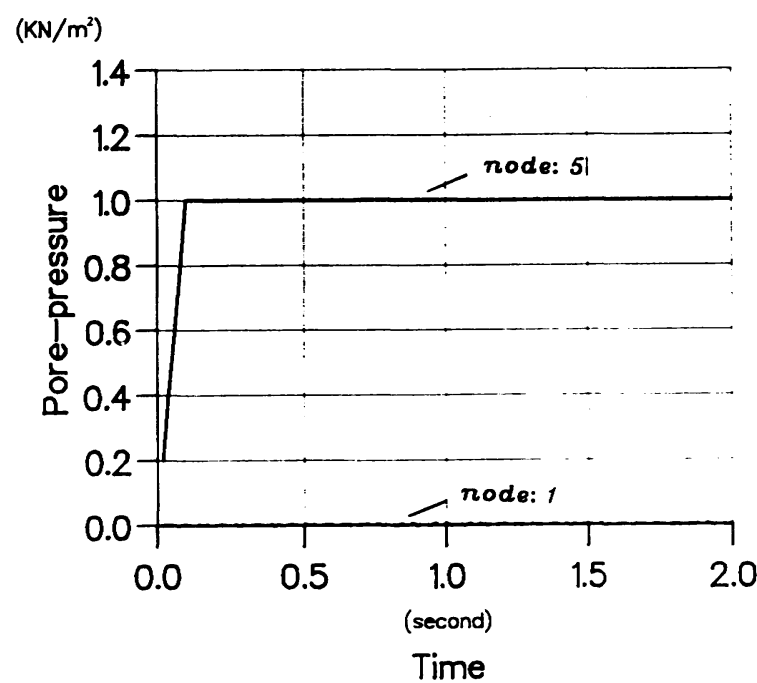

FIGURE 16 Solutions for implicit direct scheme with equal interpolation (undrained, incompressible).

ever, considerable computational cost reduction is obtained in such a large computation involving earthquake response analysis of large earth dams previously calculated by the implicit direct method of Zienkiewicz and Xie [1991].

\section{APPENDIX A: STEADY-STATE FORMULATIONS}

\section{H*-Scheme $^{*}$}

Here we consider the incompressible-undrained limit case. On the boundary $\Gamma_{t}$ for which $\tilde{\mathbf{t}}$ is the

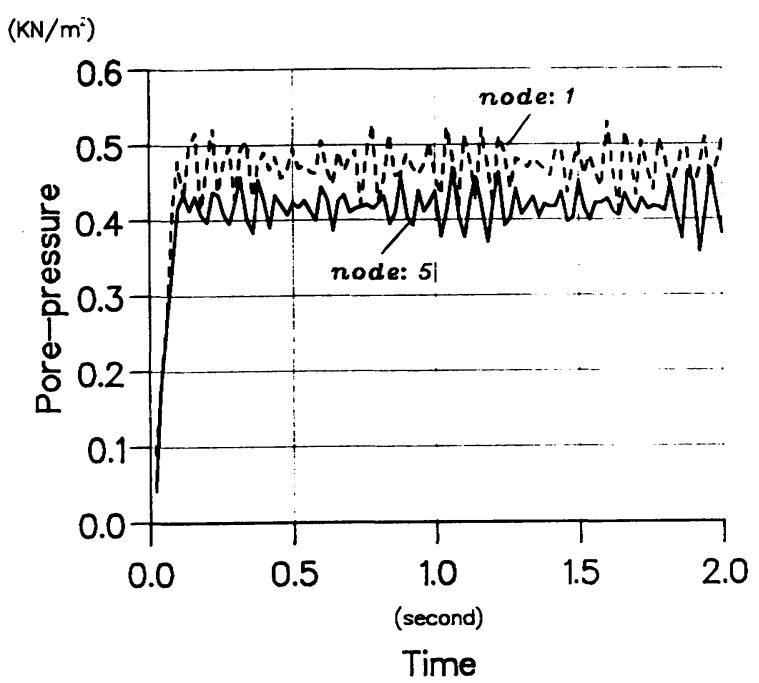

FIGURE 17 Solutions for implicit direct scheme with nonuniform interpolation (undrained, incompressible).

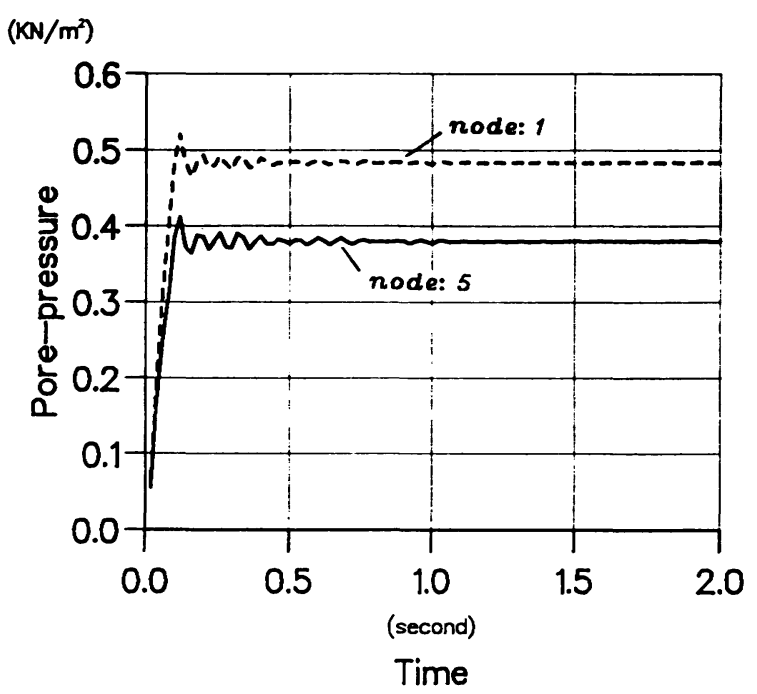

FIGURE 18 Solutions for semiexplicit $\mathbf{H}^{*}$-scheme with equal interpolation (undrained, incompressible).

prescribed traction, we take $\tilde{p}=0$. Simple linear elastic behavior is considered.

From Eqs. (16), (17), and (18), we have

$$
\begin{aligned}
& \mathbf{M}\left(\ddot{\overline{\mathbf{u}}}_{n+1}^{p}+\Delta \ddot{\overline{\mathbf{u}}}_{n}^{*}\right)+\mathbf{K} \overline{\mathbf{u}}_{n+1}^{p}-\mathbf{Q} \overline{\mathbf{p}}_{n}=\left(\mathbf{f}_{s}\right)_{n+1} \\
&\left(\mathbf{H}^{*} \theta \gamma \Delta t^{2}\right) \Delta \dot{\overline{\mathbf{p}}}_{n}=(\mathbf{A})_{n+1}-\mathbf{H}^{*} \dot{\overline{\mathbf{p}}}_{n} \gamma \Delta t^{2} \\
&-\mathbf{Q}^{T}\left(\dot{\overline{\mathbf{u}}}_{n+1}^{p}+\Delta \overline{\mathbf{u}}_{n}^{*} \gamma \Delta t\right)^{(\mathrm{A}} \\
& \mathbf{M} \Delta \ddot{\overline{\mathbf{u}}}_{n}^{* *}=\mathbf{Q}\left(\Delta t \dot{\overline{\mathbf{p}}}_{n}+\Delta \dot{\overline{\mathbf{p}}}_{n} \theta \Delta t\right)
\end{aligned}
$$

$\left(\mathrm{KN} / \mathrm{m}^{2}\right)$

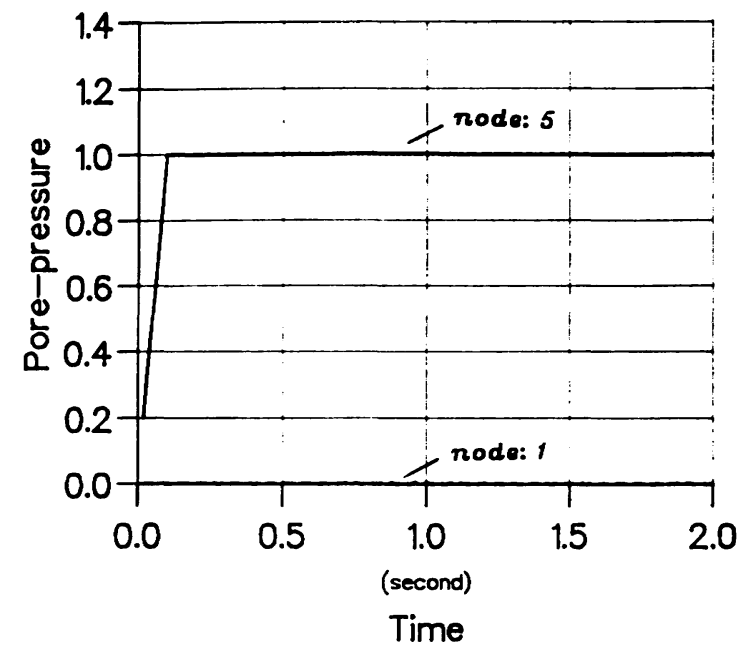

FIGURE 19 Solutions for semiexplicit $\mathbf{Q}^{T} \mathbf{M}^{-1} \mathbf{Q}$ scheme with equal interpolation (undrained, incompressible). 
where

$$
\begin{aligned}
\left(\mathbf{f}_{s}\right)_{n+1} & =\int_{\Omega} \mathbf{N}_{u}^{T} \rho \mathbf{b}_{n+1} d \Omega+\int_{\Gamma} \mathbf{N}_{u}^{T} \tilde{\mathbf{t}}_{n+1} d \Gamma \\
\mathbf{K} & =\int_{\Omega} \mathbf{B}^{T} \mathbf{D B} d \Omega .
\end{aligned}
$$

Substituting Eq. (A.1) into (A.2), we can eliminate the unknown $\Delta \ddot{\overline{\mathbf{u}}}_{n}^{*}$ and now have

$$
\begin{aligned}
& \left(\mathbf{H}^{*} \theta \gamma \Delta t^{2}\right) \Delta \dot{\overline{\mathbf{p}}}_{n}=\left(\mathbf{f}_{p}\right)_{n+1}-\mathbf{H}^{*} \dot{\overline{\mathbf{p}}}_{n} \gamma \Delta t^{2}-\mathbf{Q}^{T} \dot{\overline{\mathbf{u}}}_{n+1}^{p} \\
& -\mathbf{Q}^{T} \mathbf{M}^{-1}\left[\left(\mathbf{f}_{s}\right)_{n+1}-\mathbf{M} \ddot{\overline{\mathbf{u}}}_{n+1}^{p}-\mathbf{K} \overline{\mathbf{u}}_{n+1}^{p}+\mathbf{Q} \overline{\mathbf{p}}_{n}\right] \gamma \Delta t
\end{aligned}
$$

From Eqs. (A.1) and (A.3), we easily have

$$
\begin{aligned}
\mathbf{M}\left(\ddot{\mathbf{u}}_{n+1}^{p}+\right. & \left.\Delta \ddot{\overline{\mathbf{u}}}_{n}\right)+\mathbf{K} \overline{\mathbf{u}}_{n+1}^{p} \\
& -\mathbf{Q}\left(\overline{\mathbf{p}}_{n+1}^{p}+\Delta \dot{\overline{\mathbf{p}}}_{n} \theta \Delta t\right)=\left(\mathbf{f}_{s}\right)_{n+1}
\end{aligned}
$$

Steady state is reached when $\Delta \ddot{\overline{\mathbf{u}}}_{n}$ and $\Delta \dot{\overline{\mathbf{p}}}_{n}$ are zero and then we have immediately

$$
\mathbf{M} \ddot{\overline{\mathbf{u}}}_{n+1}^{p}+\mathbf{K} \overline{\mathbf{u}}_{n+1}^{p}-\mathbf{Q} \overline{\mathbf{p}}_{n+1}^{p}=\left(\mathbf{f}_{s}\right)_{n+1}
$$

and

$$
\begin{aligned}
\mathbf{Q}^{T} \dot{\overline{\mathbf{u}}}_{n+1}^{p} & +\mathbf{Q}^{T} \mathbf{M}^{-1}\left[\left(\mathbf{f}_{s}\right)_{n+1}-\mathbf{M} \ddot{\mathbf{u}}_{n+1}^{p}-\mathbf{K} \overline{\mathbf{u}}_{n+1}^{p}\right. \\
& \left.+\mathbf{Q} \overline{\mathbf{p}}_{n}\right] \gamma \Delta t+\mathbf{H}^{*} \dot{\overline{\mathbf{p}}}_{n} \gamma \Delta t^{2}=\left(\mathbf{f}_{p}\right)_{n+1}
\end{aligned}
$$

Substituting Eq. (A.6) into (A.7), we have

$$
\mathbf{Q}^{T} \dot{\overline{\mathbf{u}}}_{n+1}^{p}+\left(\mathbf{H}^{*}-\mathbf{Q}^{T} \mathbf{M}^{-1} \mathbf{Q}\right) \dot{\overline{\mathbf{p}}}_{n} \gamma \Delta t^{2}=\left(\mathbf{f}_{p}\right)_{n+1}
$$

or

$$
\begin{array}{r}
\mathbf{Q}^{T}\left(\dot{\overline{\mathbf{u}}}_{n}+\Delta t \ddot{\overline{\mathbf{u}}}_{n}\right)+\left(\mathbf{H}^{*}-\mathbf{Q}^{T} \mathbf{M}^{-1} \mathbf{Q}\right) \dot{\overline{\mathbf{p}}}_{n} \gamma \Delta t^{2} \\
=\left(\mathbf{f}_{p}\right)_{n+1}
\end{array}
$$

By integrating Eq. (A.9) in time, we have

$$
\mathbf{Q}^{T}\left(\overline{\mathbf{u}}_{n}+\Delta t \dot{\overline{\mathbf{u}}}_{n}\right)+\left(\mathbf{H}^{*}-\mathbf{Q}^{T} \mathbf{M}^{-1} \mathbf{Q}\right) \overline{\mathbf{p}}_{n} \gamma \Delta t^{2}=\left(\mathbf{f}_{p}^{*}\right)_{n+1}
$$

where $\left(\mathbf{f}_{p}^{*}\right)_{n+1}$ is the time integrated form $\left(\mathbf{f}_{p}\right)_{n+1}$.

Finally, when the steady state is reached, from Eqs. (A.6) and (A.10) we have

$$
\mathbf{K} \overline{\mathbf{u}}-\mathbf{Q} \overline{\mathbf{p}}=\mathbf{f}_{s}
$$

and

$$
\mathbf{Q}^{T} \overline{\mathbf{u}}+\left(\mathbf{H}^{*}-\mathbf{Q}^{T} \mathbf{M}^{-1} \mathbf{Q}\right) \overline{\mathbf{p}} \gamma \Delta t^{2}=\mathbf{f}_{p}^{*}
$$

or

$$
\left[\begin{array}{cc}
\mathbf{K} & -\mathbf{Q} \\
\mathbf{Q}^{T} & \left(\mathbf{H}^{*}-\mathbf{Q}^{T} \mathbf{M}^{-1} \mathbf{Q}\right) \gamma \Delta t^{2}
\end{array}\right]\left\{\begin{array}{l}
\overline{\mathbf{u}} \\
\overline{\mathbf{p}}
\end{array}\right\}=\left\{\begin{array}{c}
\mathbf{f}_{s} \\
\mathbf{f}_{p}^{*}
\end{array}\right\}
$$

From Eq. (A.13), we can observe that this scheme can avoid the restriction of mixed formulation.

\section{$\mathbf{Q}^{T} \mathbf{M}^{-1} \mathbf{Q}-$ Scheme}

Similarly, for $\mathbf{Q}^{T} \mathbf{M}^{-1} \mathbf{Q}$-scheme we only replace the matrix $\mathbf{H}^{*}$ with $\mathbf{Q}^{T} \mathbf{M}^{-1} \mathbf{Q}$. Following the same procedures, we can easily obtain the steady-state equation:

$$
\left[\begin{array}{cc}
\mathbf{K} & -\mathbf{Q} \\
\mathbf{Q}^{T} & \mathbf{0}
\end{array}\right]\left\{\begin{array}{l}
\overline{\mathbf{u}} \\
\overline{\mathbf{p}}
\end{array}\right\}=\left\{\begin{array}{c}
\mathbf{f}_{s} \\
\mathbf{f}_{p}^{*}
\end{array}\right\}
$$

Obviously, this scheme does not avoid the restriction of mixed formulation.

\section{REFERENCES}

Borja, R. I., 1991, "Composite Newton-PCG and Quasi-Newton Iterations for Nonlinear Consolidation," Computer Methods in Applied Mechanics and Engineering, 86, 27-60.

Chan, A. H. C., Famiyesin, O. O., and Wood, D. M., "A Fully Explicit u-w Scheme for Dynamic Soil and Pore Fluid Internation," in Cheung, Y. K. et al. Computational Mechanics, 1991, Balkema.

Katona, M. G., and Zienkiewicz, O. C., 1985, "A Unified Set of Single Step Algorithms. Part 3: The Beta-m Method, a Generalization of the Newmark Scheme," International Journal for Numerical Methods in Engineering, 21, 1345-1359.

Zienkiewicz, O. C., Chan, A. H. C., Pastor, M., Paul, D. K., and Shiomi, T., 1990, "Static and Dynamic Behaviour of Soil: A Rational Approach to Quantitative Solutions. I. Fully Saturated Problems,"' Proceedings of the Royal Society of London, A, 429, 285-309.

Zienkiewicz, O. C., Hinton, E., Leung, K. H., and Taylor, R. L., "Staggered, Time Marching Schemes in Dynamic Soil Analysis and Selective Explicit Extrapolation Algorithms," in R. Shaw et al. Proceedings of the 2nd Symposium on Innovative Analysis for the Analysis for the Engineering Sciences, 1980, University of Virginia Press. 
Zienkiewicz, O. C., and Shiomi, T., 1984, "Dynamic Behaviour of Saturated Porous Media: The Generalised Biot Formulation and Its Numerical Solution."' International Journal for Numerical and Analytical Methods in Geomechanics, 8, 71-96.

Zienkiewicz, O. C., and Wu, Jie, 1991, "Incompressi- bility Without Tears-How to Avoid Restrictions of Mixed Formulation," International Journal for $\mathrm{Nu}$ merical Methods in Engineering, 32, 1189-1203.

Zienkiewicz, O. C., and Xie, Y. M., 1991, "Analysis of the Lower San Fernando Dam Failure Under Earthquake," Dam Engineering, II, 307-322. 

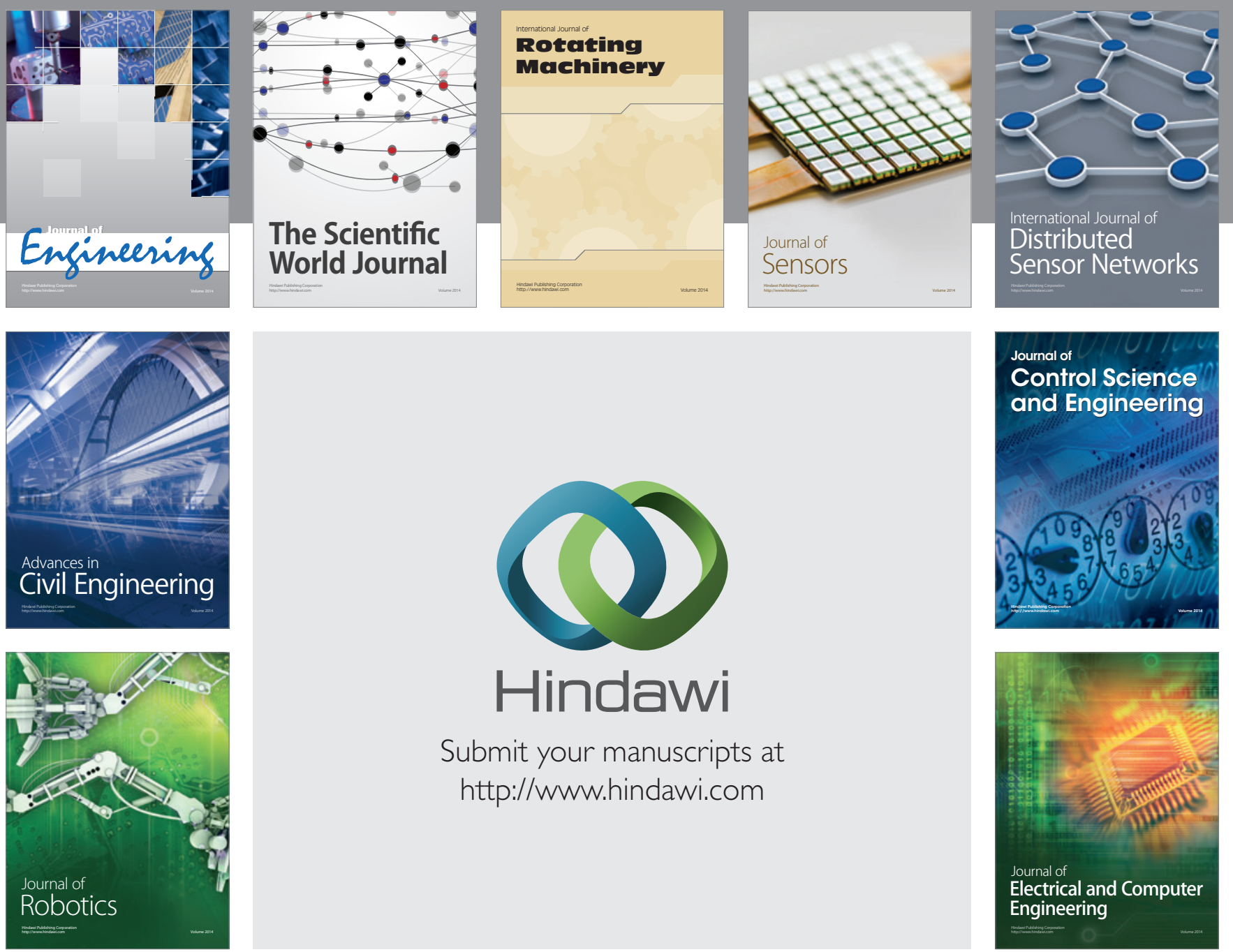

Submit your manuscripts at

http://www.hindawi.com
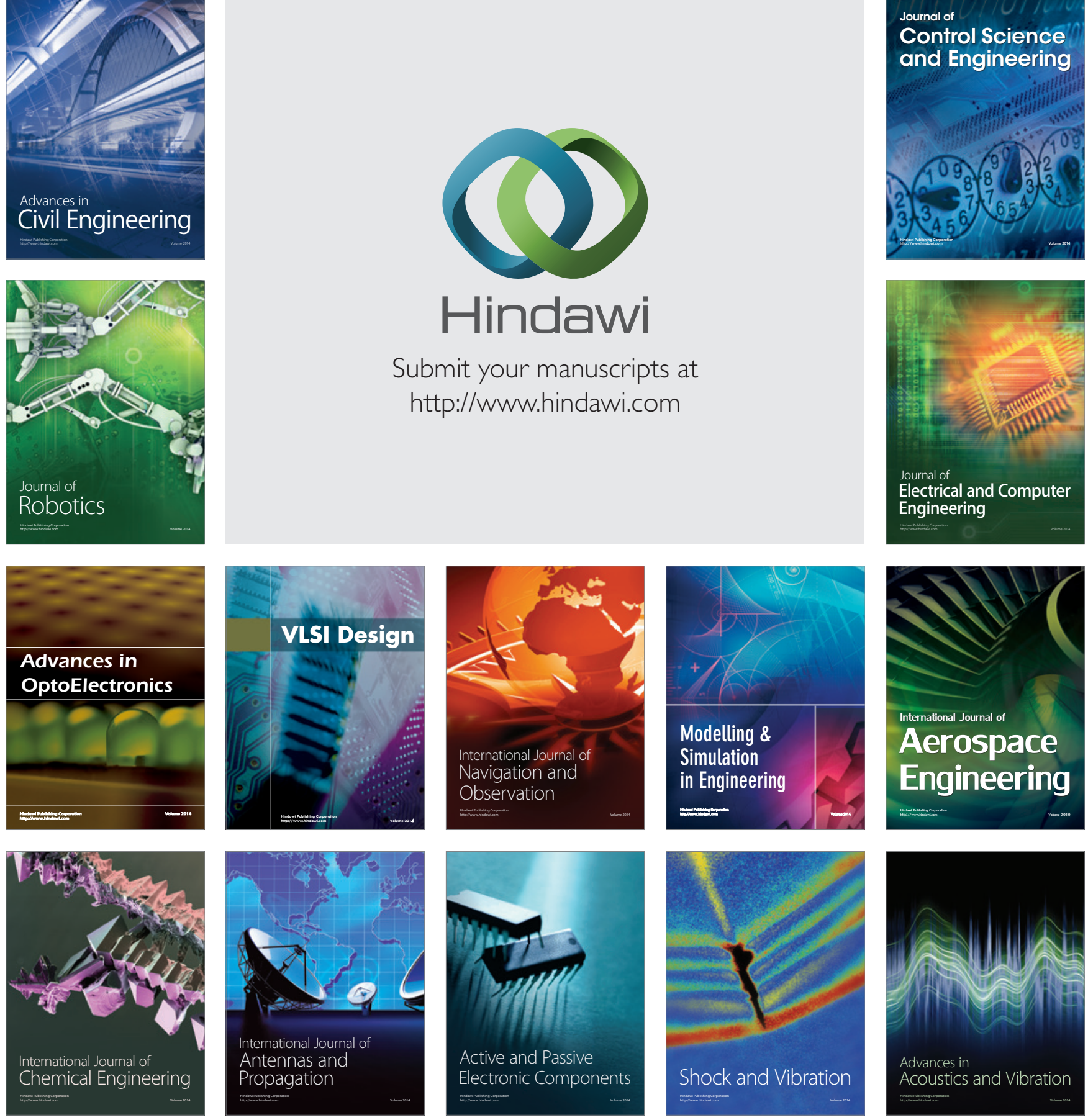